Archives

$17 \mid 1996$

Hommage à Bernard Lepetit

\title{
Le champ urbain comme discours de légitimation politique. Le cas de Mendoza (Argentine)
}

\section{Ricardo Ponte}

\section{(2) OpenEdition}

Journals

Édition électronique

URL : http://journals.openedition.org/ccrh/2590

DOI : $10.4000 /$ ccrh. 2590

ISSN : $1760-7906$

Éditeur

Centre de recherches historiques - EHESS

Édition imprimée

Date de publication : 4 octobre 1996

ISSN : 0990-9141

Référence électronique

Ricardo Ponte, «Le champ urbain comme discours de légitimation politique. Le cas de Mendoza (Argentine) », Les Cahiers du Centre de Recherches Historiques [En ligne], 17| 1996, mis en ligne le 27 février 2009, consulté le 19 avril 2019. URL : http://journals.openedition.org/ccrh/2590 ; DOI : $10.4000 /$ ccrh. 2590

Ce document a été généré automatiquement le 19 avril 2019

Article L.111-1 du Code de la propriété intellectuelle. 


\title{
Le champ urbain comme discours de légitimation politique. Le cas de Mendoza (Argentine)
}

\author{
Ricardo Ponte
}

\section{Le cadre géographique}

1 Mendoza est une ville de fondation espagnole (1561), située dans le centre-ouest de la république Argentine, au pied de la cordillère des Andes. Sa situation géographique, sur la route commerciale stratégique entre Buenos Aires, Santiago du Chili et Lima, fut déterminante.

2 Son tracé, réalisé lors de sa fondation, consistait en un quadrillage parfait, de cinq manzanas (pâtés de maison) sur cinq, avec une place centrale qui, peu après, fut déplacée et excentrée. L'évolution postérieure de la ville respecta le système du quadrillage espagnol, tout en l'adaptant à la topographie du terrain, une plaine doucement inclinée.

En 1861, un terrible tremblement de terre, dont l'épicentre se trouvait dans la ville même, ébranla trois siècles de développement de la ville coloniale, mais offrit la possibilité d'une reconstruction urbaine, régie par les nouveaux critères dominants de la deuxième moitié du XIX ${ }^{e}$ siècle.

4 Avec l'arrivée du chemin de fer, allant de Buenos Aires à Mendoza, en 1885, la ville connut une série de transformations mises en oeuvre par l'élite dirigeante du pays. La modernisation des structures productives visait à adapter la production locale, fondée jusqu'alors sur la production fourragère, à une économie vinicole, plus rentable en termes économiques. Arrivent, d'une part, les nouveaux équipements qui seront adoptés par l'industrie viti-vinicole, transformant l'économie locale et, d'autre part, les immigrés, principalement italiens et espagnols, qui provoquent un renouvellement presque total de la population locale. L'arrivée du chemin de fer coïncide avec celle des capitaux étrangers, principalement anglais, dans toute la république. Pour cela, le pays s'est 
d'abord «discipliné» puis «pacifié », avec l'extermination des Indiens-qui déterminaient les frontières intérieures du pays.

5 La province était gouvernée par une élite oligarchique, constituée par l'ancienne classe des propriétaires terriens, depuis le milieu du xix ${ }^{e}$ siècle, où avait commencé le processus d'« organisation nationale», avec une constitution et un projet libéral, concernant surtout l'aspect économique, mais avec de grandes restrictions dans le domaine politique, puisqu'on avait couramment recours à la fraude pour remporter les élections, et que l'on éliminait toute chance pour les secteurs populaires d'accéder au pouvoir. Cette oligarchie soutint des gouvernements de type autoritaire, avec néanmoins les traits formels d'un républicanisme restreint.

6 L'Argentine de la fin du XIX ${ }^{e}$ siècle s'intègre dans le marché mondial en tant que productrice de matières premières, dans une étroite dépendance commerciale par rapport à l'Empire britannique. Le pays commence à connaître une certaine prospérité, alors que l'élite dirigeante gouverne le pays grâce à une alliance des plus solides, qui rend notamment possible la stabilité politique pendant des décennies, avec la devise Ordre et progrès.

\section{La ville « barbare » et la ville « civilisée »}

7 Le tremblement de terre de 1861 fit l'effet d'un véritable cataclysme. La ville comptait une population de 8670 habitants (d'après le recensement de 1857). On estime que $70 \%$ de la population succomba durant la catastrophe, si l'on inclut la ville même et ses alentours. La population, d'après le recensement provincial suivant, 1864, fut réduite à 4457 habitants ${ }^{1}$.

8 Mendoza était une ville basse et plate, toute construite en adobe ${ }^{2}$ avec des maisons à un seul étage, bien qu'il y en eût à deux étages, avec des toits sans tuiles, quasi plats ou avec une légère pente, en raison du climat semi-désertique. La ville était dépourvue d'architecture monumentale, et les principaux édifices, par la taille et la qualité de la construction, étaient les églises. La structure de la ville était peu compacte, avec des limites mal définies entre la ville et la campagne et un aspect semi-rural.

9 L'ancienne ville de Mendoza avait eu comme centre de la vie urbaine, la Plaza Mayor de la fondation, face à laquelle se trouvait le Cabildo (municipalité), l'église Matriz, l'église de la Compagnie de Jésus, la poste, et la plus grande partie de la population. Après le tremblement de terre, le siège du gouvernement local fut déplacé vers la nouvelle ville. L'ancienne ville ne put résister à tant de catastrophes. Elle fut désarticulée fonctionnellement et, progressivement, ses usages se dégradèrent.

10 Par ailleurs, le projet de nouveau village, au sud-ouest de l'ancienne ville, était organisé sur la base d'un polygone de huit manzanas sur huit, avec comme centre une grande place-parc (de quatre manzanas). Équidistantes de celle-ci, et sur les diagonales du polygone, furent intégrées quatre autres places, créant un schéma symétrique avec le centre, dans l'intention de faire tourner la vie urbaine du nouveau village autour de la grande place centrale, à partir des nouveaux usages.

11 On conserva beaucoup de décombres de l'ancienne ville - durant plus de deux décennies. Mais autant l'ancienne (où étaient restés les secteurs les plus démunis) que la nouvelle (où s'étaient déplacés les secteurs les plus aisés) tentèrent de conserver « le » centre de la ville : l'une, par résistance passive et l'autre, par des politiques actives. 
12 La reconstruction après le tremblement de terre offrit une occasion unique de façonner une « nouvelle ville » moderne, en opposition avec l'ancienne ville, de caractère espagnol et qui, comme tout ce qui était espagnol, était associée par l'oligarchie libérale dirigeante à l'arriération du pays. Cette dernière se proposa délibérément de faire, non seulement une "nouvelle ville», mais encore, une "nouvelle société", comme l'avait exprimé devant l'assemblée législative locale, le jeune gouverneur Carlos González Pinto ${ }^{3}$.

Par ailleurs, à partir de la référence au lointain modèle de Paris, et de celui, plus proche, de Buenos Aires, on commençait à valoriser la présence du large boulevard planté d'arbres. À Mendoza, ce «type urbain » existait déjà avec l'avenue San Martin. Il fallait seulement l'institutionnaliser et le mettre en valeur, ce qu'on fit finalement. La rue qui avait constitué la limite de la ville coloniale, où était située l'unique Alameda (allée plantée d'arbres) maintenue comme promenade dans l'Argentine actuelle (qui marquait la limite de cette ville après le tremblement de terre), articula les deux villes qui devinrent finalement une seule du point de vue de la morphologie urbaine, même si les différences sociales qui les marquaient dès le début avaient été maintenues.

Ces circonstances, si particulières au développement historique de Mendoza, ont donné à ses caractéristiques urbaines une configuration inédite parmi les villes argentines, la vie urbaine principale étant située le long d'une rue, et non autour d'une place principale.

\section{La modernité comme processus de confrontation entre les élites}

Avec l'organisation du pouvoir politique dans les mains d'une élite oligarchique, sous le contrôle d'un chef "naturel», Emilio Civit, la province de Mendoza s'apprêta à réorganiser la ville capitale et la province, en prévision des temps nouveaux. Les exclusions conjoncturelles du pouvoir politique dominant, vis-à-vis de l'ancienne élite locale, engendraient un espace pour les luttes intra-oligarchiques qui se manifestaient principalement dans le domaine des représentations sociales, mais aussi dans les domaines politique, économique et culturel.

Sur le terrain politique, la constitution provinciale fut réformée, dans le but d'assurer la prééminence du pouvoir exécutif sur le législatif. La concentration grandissante du pouvoir dans les mains du gouverneur fit que, bien que la ville comptât avec une municipalité et un chef municipal, ce fut en réalité le gouverneur qui intervenait directement sur l'avenir de la ville, prenant les décisions qui concernaient les projets et le futur.

17 Ce groupe dirigeant n'envisagea aucune politique urbaine particulière pour le tracé urbain qui avait été proposé, en 1863, à l'occasion du projet de nouveau village, après le tremblement de terre. En réalité, on le respecta et on encouragea le remplissage et la densification du tracé urbain, qui paraissait encore trop ample pour la ville à la fin du siècle. L'architecture urbaine était dépourvue d'édifices monumentaux et, à l'exception d'un projet d'emplacement pour la maison du gouvernement, au centre de la place Independencia-qui n'a pas abouti-, on n'assigna à l'architecture aucun rôle d'organisation de l'espace urbain, ni même de référent spatial déterminant.

18 En revanche, le projet officiel de la période, plus ambitieux, fut la réalisation d'un parc artificiel de 300 hectares, situé entre le centre de la ville et les premiers chaînons (tout proches) des montagnes, et la création de tout un système d'irrigation avec canaux et 
acequias. Cette initiative concentra tous les efforts de l'homme politique le plus puissant de la période, et la figure la plus emblématique de cette oligarchie provinciale, Emilio Civit ${ }^{4}$.

L'accès à la présidence de la République du général Julio A. Roca fut l'expression d'un grand accord entre l'oligarchie porteña $a^{5}$ et les oligarchies provinciales. Grâce à cette hégémonie réussie, Emilio Civit accède à un ministère national (celui des Obras y Servicios públicos), même si cela l'oblige à renoncer au poste de gouverneur de la province de Mendoza (1898), quelques mois seulement après sa première prise de fonctions.

Étant donné que la Constitution argentine de l'époque ne permettait pas la réélection immédiate du président Roca, le problème de cette succession se posait à Civit, qui aspirait à être le candidat du parti de Roca. Néanmoins, le vœu d'Emilio Civit ne fut pas exaucé, et il dut retourner dans sa province natale pour être élu à nouveau gouverneur. Cette deuxième occasion de Civit lui offre la possibilité de faire preuve d'une gestion digne d'un présidentiable. D'où l'affichage d'un cosmopolitisme dans la recherche de ses collaborateurs. Ainsi, Mendoza devint un laboratoire d'expérimentations sur la manière de gérer le pouvoir et de montrer ses capacités à se mettre "à la hauteur des temps", dans la capitale de la province de Cuyo ${ }^{6}$.

Le choix d'un grand parc public comme œuvre symbolique de son administration peut être expliqué par diverses raisons :

a) dans une région comme Mendoza, au climat semi-désertique, la population valorise de manière exagérée la végétation, qui est toujours culturelle étant donné que la végétation naturelle est de type xérophile ;

b) dans une région sismique, on peut comprendre que, voulant faire perdurer l'œuvre audelà des contingences temporelles, on ait préféré porter son choix sur un parc plutôt que sur un bâtiment. Le tremblement de terre de 1861 détruisit les bâtiments, mais laissa intacts les arbres ;

c) un parc rend compte d'un certain apprivoisement de la nature. Ce qui s'accorde avec la conception d'un paysagisme d'orientation pittoresque à la mode en Europe qui se proposait d'embellir la nature ;

d) cela visait également à exprimer le raffinement de l'élite dirigeante de Mendoza. Disposer d'un endroit où celle-ci pouvait se promener en voiture était toute une nouveauté à l'époque. Un lieu pour voir et être vu, accomplissant le rite provincial du contact social. Mendoza était dépourvue d'aristocratie, mais on savait que l'aristocratie usait des parcs européens comme de lieux de rencontre et de réunion et les mettait en valeur.

Comme on peut le constater, la référence à Paris, comme centre de légitimation, n'est pas une idée inventée par l'observateur contemporain, qui connaît l'influence qu'eut la VilleLumière, après les réformes du baron Haussmann. C'est au contraire une référence constante et explicite qui revient sans cesse dans les journaux d'opposition. En effet, il semble curieux que la presse d'opposition prétende ridiculiser Civit en faisant référence

[...] à ses efforts patriotiques pour faire de Mendoza un Paris en miniature, avec ses bois verts de trois cents hectares?

À une autre occasion, on signale que :

La réalisation des immenses bois projetés, avec tous leurs accessoires qui, d'après les désirs de monsieur le Ministre [se référant à Civit], seront quelque chose comme les Champs-Élysées ou le bois de Boulogne, et au moins un Palermo porteño ou une Quinta Normal de Agricultura [Chili] ${ }^{8}$. 
Nous pouvons remarquer que le fait de signaler Paris comme modèle, et non la capitale du pays, Buenos Aires, a des fondements historico-politiques et culturels. La ville de Mendoza (1561) est plus ancienne que la capitale Buenos Aires (1580). Mendoza est l'une des provinces fondatrices de ce qu'on appelait alors les Provinces Unies du Río de La Plata, aujourd'hui république Argentine, mais l'organisation nationale argentine tardive fit émerger en Mendoza non pas le sentiment d'atteindre une parité réelle avec Buenos Aires, mais celui d'être considérée comme l'égale de la capitale du pays.

Le cas de la conception du parc public de Mendoza est exemplaire de cette autonomie. Son promoteur, Emilio Civit, se rendit à Paris en 1889. En 1895, on fit appel à un médecin hygiéniste français afin d'établir un diagnostic sur l'état hygiénico-sanitaire de la ville et, finalement, en 1896, on invita un architecte français, Charles Thays, pour élaborer le projet de parc. Ces deux personnalités, dans leur carrière professionnelle, étaient d'abord passées par Buenos Aires, mais c'est une chose sur laquelle on n'a pas trop insisté, à l'inverse de leur filiation parisienne.

Le fait que la presse se réfère directement à Paris, en omettant un échelon, naturellement prévisible pourrait-on dire, qui serait la capitale argentine, orientée à la même époque vers des entreprises similaires, rend compte du rôle où désirait se situer Mendoza dans l'ensemble des villes argentines de l'époque. Nous pensons que, pour le gouvernement comme pour l'opposition, il ne fait aucun doute que le modèle est Paris, même s'il constitue le référent non explicité du discours officiel. La presse sait où adresser ses critiques dans la référence au modèle.

Bien que le nom officiel du parc fût : «Parc public de l'Ouest », on savait qu'il était peu probable que les secteurs populaires en fissent usage, en raison de plusieurs inconvénients. Il était situé loin de la ville. Faute de transports en commun, il fallait s'y rendre en voiture ou même à pied. Même s'il n'y avait aucune interdiction officielle pour son utilisation, le contrôle social rigide garantissait à lui seul l'usage, par chaque secteur social, de ses lieux propres, sachant où il pouvait et où il ne pouvait pas se rendre.

S'il restait encore des doutes sur l'usage restrictif du parc, Civit fit mettre en 1909, à son entrée, des portails monumentaux de fer forgé, importés d'Angleterre, dont les portes principales n'étaient ouvertes que pour le passage du gouverneur. Ces portails remplissent la fonction symbolique de prévenir qu'il existe une entrée, et qu'il faut demander la permission pour s'y introduire, bien qu'il n'y ait pas de gardes pour interdire l'accès.

Emilio Civit ne choisit pas de réaliser n'importe quelle œuvre mais un parc. L'idée de laisser l'héritage symbolique d'un parc séduit. Les «seigneurs » de l'Antiquité classique ou de la Renaissance offraient en héritage à la communauté les parcs et jardins qu'ils avaient créés ou financés avec leurs propres moyens. Il existe dans le monde occidental, en effet, une très longue tradition, celle de lier les jardins et les parcs à la magnificence des seigneurs. Les jardins de la période humaniste s'ouvrent au grand public, comme une manière de montrer la magnificence de la famille ou de sa culture. La splendeur du seigneur et le prestige du propriétaire s'élèvent avec l'accroissement du nombre de visiteurs ${ }^{9}$.

Dans la Mendoza contemporaine, on ressent «le Parc» comme un don qu'aurait fait Emilio Civit à la ville, et non comme une réalisation publique réalisée avec le budget et les impôts de la province. Cette représentation sociale est renforcée par la construction sémantique politique, élaborée aussi bien par des personnalités locales que par des 
étrangers. Par manque d'informations ou par volonté politique délibérée, ils attribuent la création et la consolidation de cette entreprise publique grandiose, qui a pris plusieurs décennies, et qui a donc été soutenue par d'autres gouvernements, presque exclusivement à la gestion de Civit.

Nous pensons que dans la Mendoza de la fin du XIX siècle et du début du XX ${ }^{\mathrm{e}}$, l'oligarchie qui décide de l'avenir de la province de 1873 à 1918 met en œuvre une opération de légitimation d'elle-même. Le discours est surtout fondé sur l'action urbaine des administrations de la période, avec, comme œuvre emblématique, la réalisation du Parc public de l'Ouest. Il faut, en outre, remarquer que ce processus de légitimation, entrepris par les administrations conservatrices de Mendoza fut une réussite. Cela est dû, a posteriori, à l'apparition de véritables mythes, qui se réfèrent toujours à des questions urbaines élaborées dans cette période.

L'élément central, sans doute, de ce processus de légitimation fut l'exemple du Parc de l'Ouest. Ce processus particulier de construction historique relève en général du mythe, dont l'une des fonctions consiste précisément à donner une idée simplifiée, compréhensible et fermée des faits historiques.

Les dirigeants conservateurs locaux se servirent de Civit pour construire la figure du « héros civique » qui aurait fondé la « Mendoza moderne ». Pour cette raison, ce discours de légitimation de la classe conservatrice de Mendoza est également, à son tour, un discours « fondateur ». D'une manière ou d'une autre, on a effacé, dans le présent, toutes les traces des critiques qui avaient été faites, à l'époque, contre Civit, gouverneur de la province. Toute l'opposition de la presse, toutes les accusations, y compris celles liées à la corruption, ont disparu de la mémoire collective locale, et il ne reste que « les traces du bienfaiteur ». Pour compléter ce tableau hégémonique, la version contemporaine que l'on connaît sur tous ces événements et sur toute l'œuvre de Civit, a recours à une seule et unique source : l'historiographie « civitiste».

Lorsqu'on lit, dans la partie consacrée au Parc de l'Ouest de l'Album argentino (1909), édité en hommage à Emilio Civit, vers la fin de son deuxième mandat comme gouverneur de Mendoza :

Tant que Mendoza aura son parc, Civit gardera son prestige, on comprend clairement quel fut l'objectif symbolique que Civit lui-même avait attribué à ce parc artificiel et exotique, sur le piémont de Mendoza.

\section{NOTES}

1. R. Ponte, Mendoza, aquella ciudad de barro. Historia de una ciudad andina desde el siglo XVI hasta nuestros días, Ville de Mendoza, 1987, p. 160 et 538.

2. L'adobe est une terre, cuite au soleil et mélangée avec de la paille, utilisé de préférence sur des zones à faibles précipitations, dans les climats désertique ou semi-désertique.

3. José Luis Massini Calderon, Mendoza hace cien años, Mendoza, Ed. Teoría, 1967, p. 187.

4. Cet homme politique était le fils d'un ancien gouverneur. Il avait occupé lui-même tous les postes possibles dans la hiérarchie de l'administration publique provinciale et nationale : depuis 
le poste de député et sénateur national, jusqu'à celui de président de la municipalité ; depuis le poste de ministre, provincial et national, jusqu'à celui de gouverneur. Réélu en tant que gouverneur, il contrôla, soit directement au pouvoir, soit dans ses coulisses, tout le quart de siècle qui s'étend de 1885 à 1910, année qui marque la fin de son deuxième mandat en tant que gouverneur et que l'on considère comme le point culminant de toute cette génération.

5. En Argentine, le terme porteño désigne la population de Buenos Aires, faisant ainsi allusion à la fonction traditionnelle de port.

6. La province de Cuyo, d'après la division politico-administrative de 1776 avec la création de la vice-royauté du Río de La Plata, et jusqu'en 1820, avait pour capitale la ville de Mendoza. Le libertador, le général San Martín, "père de la patrie argentine », fut précisément gouverneurintendant de la province de Cuyo, d'où il prépara son armée pour l'indépendance du Chili et du Pérou. Le terme "Cuyo » est d'origine indigène et signifierait: "Terre des sables ». Par extension, l'adjectif qui en dérive, cuyano, renvoie aux anciennes provinces de Mendoza, San Juan et San Luis.

7. Journal Los Andes, Mendoza, 27 septembre 1896.

8. Ibid., 29 janvier 1897.

9. F. Panzini, «Per i piacere del popolo. La Evoluzione del giardino pubblico », Europa dalle origini al XX secolo, Bologne, Zanichelli, 1996, p. 25.

\section{AUTEUR}

\section{RICARDO PONTE}

Architecte, université de Mendoza (Argentine), 1975. Spécialisation en restauration de monuments, université Studi, Florence, 1978. DESAL, Institut des hautes études de l'Amérique latine, université de Paris III, 1995.

EHESS, sous la direction de Bernard Lepetit : Doctorant.

Chercheur au Centro regional de investigaciones científicas y tecnológicas (CRICYT), Mendoza (Argentine). 